\title{
Hardy's entanglement as the ultimate explanation for the observed cosmic dark energy and accelerated expansion
}

\author{
Mohamed S. El Naschie \\ Dept. of Physics, University of Alexandria, Alexandria, Egypt
}

Email address:

chaossf@aol.com

To cite this article:

Mohamed S. El Naschie. Hardy's Entanglement as the Ultimate Explanation for the Observed Cosmic Dark Energy and Accelerated Expansion. International Journal of High Energy Physics. Vol. 1, No. 2, 2014, pp. 13-17. doi: 10.11648/j.ijhep.20140102.11

\begin{abstract}
We reason that Hardy's probability of quantum entanglement marks the transition from a smooth 4D to a rugged fractal-like K3 Kähler spacetime. The associated eigenvalue constituting the measurable ordinary energy density in this case is given by Einstein's celebrated formula $\mathrm{E}=\mathrm{mc}^{2}$ divided by 22 where $\mathrm{m}$ is the mass and $\mathrm{c}$ is the speed of light. That way the missing energy is concluded to be a hypothetical so called dark energy amounting to $E(D)=E-E(O)$ where $E(O)$ is the earlier mentioned measurable ordinary energy. By looking deeper at the nature of $\mathrm{E}(\mathrm{O})$ and $\mathrm{E}(\mathrm{D})$ components of $\mathrm{E}(\mathrm{Einstein)}$ it becomes evident that $\mathrm{E}(\mathrm{O})$ is a quasi potential energy of the quantum particle modeled by the zero quantum set while $\mathrm{E}(\mathrm{D})$ is a quasi kinetic energy of the propagating quantum wave as modeled by the empty quantum set of our transfinite quantum set theory. A particularly highly interesting new result of the present work is a demonstration of the independence of dark energy density from the number of the spacetime dimensions of the corresponding theory used.
\end{abstract}

Keywords: Accelerated Cosmic Expansion, Dark Energy, Hardy's Quantum Entanglement, Superstrings, Ricci Dark Energy, Holographic Principle, 'tHooft-Veltman-Wilson Dimensional Regularization

\section{Introduction}

In numerous previous publications [1-37] the major problem of accelerated cosmic expansion and the associated puzzle of the missing $95.5 \%$ dark energy density of the cosmos was considered [38-44] from a host of different view points using various spacetime theories [1-37]. Thus superstrings, M-theory, holographic principle, Ricci dark energy, Rindler space, fuzzy K3 Kähler, Veneziano model, varying speed of light, Cosserat spacetime and 'tHooft-Veltman-Wilson dimensional renormalization [1] were applied leading to a robust result, namely $\mathrm{E}$ (ordinary) = $\mathrm{mc}^{2} / 22, \mathrm{E}($ dark $)=\mathrm{mc}^{2}(21 / 22)$ and $\mathrm{E}=\mathrm{E}($ ordinary $)+\mathrm{E}($ dark $)$ $=\mathrm{mc}^{2}=\mathrm{E}($ Einstein $)$ where $\mathrm{m}$ is the mass and $\mathrm{c}$ is the speed of light in full agreement with cosmological observations, measurement and analysis [37-42].

In the present work we pay special attention to revisiting an earlier result showing the role of Hardy's quantum entanglement [45-47] in deriving ordinary energy and thus indirectly dark energy $[1,2]$. We amply demonstrate that the so obtained result is independent of the number of topological spacetime dimensions of the used theory [1-37].

\section{Analysis}

\subsection{The Kähler Manifold}

The K3 Kähler is well known from the theory of compactified extra dimensions [39]. As a measure for the ruggedness of such a manifold an index called Betti number $b_{2}^{-}$is utilized as an excellent mathematical characterization devise [39]. For a continuous smooth manifold such a Einstein's spacetime we have $b_{2}^{-}=1$. For $\mathrm{K} 3$ on the other hand which is a convoluted manifold with 4 complex dimensions $\mathrm{b}_{3}^{-}$which counts mainly the holes in this manifold, the value is considerably higher, namely $\mathrm{b}_{3}^{-}=$ 22 [39]. It is not particularly difficult to see that $\lambda=1 / b_{2}^{-}=$ $1 / 22$ may be thought of as a Weyl scaling factor transferring the Eigenvalue $\mathrm{E}=\mathrm{mc}^{2}$ of Einstein's special relativity to $\mathrm{E}($ ordinary energy $)=\left(\mathrm{mc}^{2}\right)(\lambda)=\mathrm{mc}^{2} / 22$ which marks the smooth-non-smooth boundary. This boundary in turn is a rather good approximation to the non-fractal-fractal phase transition of spacetime [33]. Pondering these results deeper still we will notice that for a fuzzy K3 Kähler we have $b_{2}^{-}$ $=1 / 22.18033989=\phi^{5} / 2$ where $\phi^{5}$ is Hardy's probability 
for the quantum entanglement of two quantum particles. It follows then that $\mathrm{E}(\mathrm{O})=\left(\phi^{5} / 2\right)\left(\mathrm{mc}^{2}\right)=\mathrm{mc}^{2} /(22.180334)$ represents an entanglement energy of a single quantum particle. Consequently the complimentary Legendre transformation of $\mathrm{E}(\mathrm{O})$ gives the disentangled major part of the energy, namely the $95.5 \%$ so called missing dark energy

$$
E(D)=1-E(O)=\left(5 \frac{\phi^{2}}{2}\right)\left(m c^{2}\right)=m c^{2}\left(\frac{21.8033989}{22.18033989}\right)
$$

\subsection{Set Theoretical Interpretation of the Result}

It is not difficult to derive the preceding ordinary energy relation

$$
\mathrm{E}(\mathrm{O})=\mathrm{mc}^{2}\left(\phi^{5} / 2\right)
$$

from E-infinity quantum set theory and find out that the zero set in five intersectional dimensions give us the rest energy of the quantum particle as the familiar measurable ordinary energy [1-37]. On the other hand $E(D)$ is equally easy to derive as the empty set in five additive dimensional space leading to the propagation kinetic energy of the quantum wave [1-37]. Since measurement leads to wave collapse, the energy could not possibly be measured at present unless future development leads to wave collapse free measurement instruments [1-37]. Consequently the wave energy remains unmeasurable and is therefore dubbed dark energy. It is needless to reiterate that this entire situation, i.e. the splitting of Einstein's $\mathrm{E}=\mathrm{mc}^{2}$ into two parts takes place only near to the Planck and Hubble length i.e. the extremely small and extremely large length scale and that for almost all other situations, $\mathrm{E}=\mathrm{mc}^{2}$ is highly accurate $[19,20]$.

\subsection{The Independence of $E(O)$ and $E(D)$ from the Number of the Topological Dimensions of the Used Theory - Quantum Entanglement Interpretation}

Hardy's quantum entanglement is one of the most important results in quantum physics which stands on firm theoretical as well as experimental ground. It is also the main door from which the famous transfinite number $\phi=2 /(1+\sqrt{5})$ enters into fundamental physics. Simply stated the maximal quantum probability of two quantum particles is given by Hardy's value [45-47]

$$
\mathrm{P}(\text { Hardy })=\phi^{5} \text {. }
$$

In E-infinity theory it is shown that this value consists of two parts, namely $\mathrm{P}_{1}=\phi^{3}$ which is termed the counter factual part while $\mathrm{P}_{1}=\phi^{n}$ is the local part where $\mathrm{n}$ is the number of the entangled quantum particles. From the above it follows that $\mathrm{P}=\phi^{5}$ could be looked upon as a topological maximal energy, i.e. a topological Planck energy binding two entangled quantum particles together. Thus the share of each particle is clearly a contribution equal to [47]

$$
\mathrm{E}(\text { topological })=\phi^{5} / 2
$$

We now come to a subtle point which requires us to be open minded and imaginative in applying Emmy Noether's celebrated theorem and the associated flexible exchange of essentially identical basic concepts, namely probability, particles and dimensions. Proceeding in this way we can interpret the pre-particle Hardy entanglement $\phi^{5} / 2$ as a reduction per each topological dimension. Thus an integer topological dimension $\mathrm{D}_{\mathrm{T}}=1$ transmutes to a corresponding "shrunk" quasi Hausdorff fractal dimension equal to

$$
D_{H}=D_{T}-\left(\phi^{5} / 2\right)=1-\left(\phi^{5} / 2\right)=5 \phi^{2} / 2 \text {. }
$$

Consequently for an $\mathrm{n}$ dimensional theory we have $(\mathrm{n})(5$ $\left.\phi^{2} / 2\right)$. The resulting quasi fractal space dimension is consequently

$$
n-(n)\left(5 \phi^{2} / 2\right)=n\left[1-\left(\phi^{5} / 2\right)\right] .
$$

Evidently this fixes our scaling factor $\lambda$ to [1-37]

$$
\lambda=\frac{n\left[1-\left(\phi^{5} / 2\right)\right]}{n}=1-\left(\phi^{5} / 2\right)=5 \phi^{2} / 2 \cong 21 / 22 .
$$

Thus our dark energy density is independent of $\mathrm{n}$ and agrees completely with our earlier result [1-37].

\section{Conclusion}

Ultimately the "mechanical" explanation for the existence of dark energy lies in effect in Hardy's celebrated theorem for two entangled quantum particles as given by $\phi^{5}$ where $\phi=2 /(1+\sqrt{5})$. This effect becomes indirectly noticeable and splits Einstein's formula $\mathrm{E}=\mathrm{mc}^{2}$ into two parts, an ordinary and a dark part only at extremely large distances near what appears to be the holographic boundary of the holographic projection of the universe. This projection is modeled by extraordinary precision via the compactified Klein modular curve of E-infinity theory. At such ultra large distances and by Witten's T-duality at the ultra short Planck distances, spacetime undergoes a fractal phase transition marked by a scaling exponent $\lambda$ equal to $21 / 22$ which is equal to the measured dark energy density of the cosmos

$$
\mathrm{E}(\mathrm{D})=\mathrm{E}(\text { Einstein })(\lambda)=\left(\mathrm{mc}^{2}\right)(21 / 22) \text {. }
$$

Since measurement collapses the Hartle-Hawking quantum wave of the universe, this "dark" energy cannot be measured using present measurement technology. The present analysis, as in previous ones, could not have been possible without relying on the techniques and methodology of the fractal-Cantorian spacetime theory developed by G. Ord, L. Nottale and M.S. El Naschie [51-71]. An excellent introduction to fractals and E-infinity quantum spacetime mingling popular scientific writing with mathematical rigor may be found in the Journal of the Institute of Science in Society by Dr. Mae-Wan Ho [72-77]. 


\section{References}

[1] M.S. El Naschie, Cosmic dark energy from 't Hooft's dimensional regularization and Witten's topological quantum field pure gravity. J of Quantum Information Science, 4, 2014, pp. 83-91.

[2] M.S. El Naschie, The meta energy of dark energy. Open Journal of Philosophy. 4, 2014, pp. 157-159.

[3] M.S. El Naschie, Entanglement of E8E8 exceptional Lie symmetry group dark energy, Einstein's maximal total energy and the Hartle-Hawking no boundary proposal as the explanation for dark energy. World Journal of Condensed Matter Physics, 4, 2014, pp. 74-77.

[4] M.S. El Naschie, Calculating the exact experimental density of the dark energy in the cosmos assuming a fractal speed of light. Int. Journal of Modern Nonlinear Theory \& Application, 3, 2014, pp. 1-5.

[5] M.S. El Naschie, Pinched material Einstein space-time produces accelerated cosmic expansion. Int. Journal of Astronomy and Astrophysics, 4, 2014, pp. 80-90.

[6] L. Marek-Crjac, Ji-Huan He, An invitation to El Naschie's theory of Cantorian space-time and dark energy. Int. Journal of Astronomy and Astrophysics, 3, 2013, pp. 464-471.

[7] M.S. El Naschie, From Yang-Mills photon in curved spacetime to dark energy density. Journal of Quantum Information Science. 3, 2013, pp. 121-126.

[8] M.A. Helal, L. Marek-Crnjac, Ji-Huan He, The three page guide to the most important results of M.S. El Nashie's research in E-infinity quantum physics and cosmology. Open Journal of Microphysics, 3, 2013, pp. 141-145.

[9] L. Marek-Crnjac, M.S. El Naschie, Quantum gravity and dark energy using fractal Planck scaling. Journal of Modern Physics, 4, 2013, pp. 31-38.

[10] M.S. El Naschie, Capillary surface energy elucidation of the cosmic dark energy - ordinary energy duality. Open Journal Fluid Dynamics, 4, 2014, 15-17.

[11] M.S. El Naschie, A Rindler-KAM spacetime geometry and scaling the Planck scale solves quantum relativity and explains dark energy. Int. Journal of Astronomy and Astrophysics, 3, 2013, pp. 483-493.

[12] M.S. El Naschie, The hydrogen atom fractal spectra, the missing dark energy of the cosmos and their Hardy quantum entanglement. Int. Journal of Modern Nonlinear Theory \& Application, 2, 2013, pp. 167-169.

[13] M.S. El Naschie, What is the missing dark energy in a nutshell and the Hawking-Hartle quantum wave. Int. Journal of Astronomy and Astrophysics, 3, 2013, pp. 205-211.

[14] M.S. El Naschie, Nash embedding of Witten's M-theory and the Hawking-Hartle quantum wave of dark energy. Journal of Modern Physics. 4, 2013, pp. 1417-1428.

[15] M.S. El Naschie, Atef Helal, Dark energy explained via the Hawking-Hartle Quantum wave and the topology of cosmic crystallography. Int. Journal of Astronomy and Astrophysics, 3, 2013, pp. 318-343.

[16] M.S. El Naschie, The missing dark energy of the cosmos from light cone topological velocity and scaling of the Planck scale. Open Journal of Microphysics, 3, 2013, pp. 64-70.

[17] L. Marek-Crnjac, M.S. El Naschie, Chaotic fractal tiling for the missing dark energy and Veneziano model. Applied Mathematics, 4, 2013, pp. 22-29.

[18] M.S. El Naschie, Dark energy from Kaluza-Klein spacetime and Noether's theorem via Lagrangian multiplier method. Journal of Modern Physics, 4, 2013, pp. 757-760.

[19] M.S. El Naschie, Quantum entanglement, Where dark energy and negative gravity plus accelerated expansion of the universe comes from. Journal of Quantum Information Science, 3, 2013, pp. 57-77.

[20] L. Marek-Crnjac, M.S. El Naschie, Ji-Huan He, Chaotic fractals at the relativistic quantum physics and cosmology. Int Journal of Modern Nonlinear Theory \& Applications, 2, 2013, pp. $78-88$.

[21] M.S. El Naschie, A fractal sponge space-time proposal to reconcile measurements and theoretical predictions of cosmic dark energy. Int. Journal of Modern Nonlinear Theory \& Applications, 2, 2013, pp. 107-121.

[22] M.S. El Naschie, A resolution of cosmic dark energy via a quantum entanglement relativity theory. Journal of Quantum Information Science, 3, 2013, pp. 23-26.

[23] M.S. El Naschie, Topological-geometrical and physical interpretation of the dark enegy of the cosmos as a 'halo' energy of the Schrödinger quantum wave. Journal of Modern Physics, 4, 2013, pp. 591-596.

[24] M. S. El Naschie, A Unified Newtonian-Relativistic Quantum Resolution of the Supposedly Missing Dark Energy of the Cosmos and the Constancy of the Speed of Light. Int. J. of Mod. Nonlinear Theory \& Applications, 2(1), 2013, pp. 43-54.

[25] M.S. El Naschie, L. Marek-Crnjac, Deriving the exact percentage of dark energy using a transfinite version of Nottale's scale relativity. Int. Journal of Modern Nonlinear Theory \& Applications, 1, 2012, pp. 118-124.

[26] Ji-Huan He, L. Marek-Crnjac, Mohamed El Naschie's revision of Albert Einstein's $\mathrm{E}=\mathrm{m}_{0} \mathrm{c}^{2}$, A definite resolution of the mystery of the missing dark energy of the cosmos. Int. Journal of Modern Nonlinear Theory \& Applications, 2, 2012, pp. 55-59.

[27] M.S. El Naschie, S. Olsen, Ji-Huan He, S. Nada, L. Marek-Crnjac, A. Helal, On the need for fractal logic in high energy quantum physics. Int. Journal of Modern Nonlinear Theory \& Applications, 2, 2012, pp. 84-92.

[28] M. S. El Naschie, The hyperbolic Extension of Sigalotti-Hendi-Sharifzadeh's Golden Triangle of Special Theory of Relativity and the Nature of Dark Energy. J. of Mod. Phys., Vol. 4, No. 3, 2013, pp. 354-356.

[29] M.S. El Naschie, Quantum entanglement as a consequence of a Cantorian micro spacetime geometry. J. of Quantum Info. Sci., Vol. 1, No. 2, 2011. pp. 50-53.

[30] M.S. El Naschie, Einstein's general relativity and pure gravity in a Cosserat and De Sitter-Witten spacetime setting as the explanation of dark energy and cosmic accelerated expansion. Int. Journal of Astronomy and Astrophysics, 4, 2014, pp. 332-339. 
[31] M.S. El Naschie, Deriving $E=\mathrm{mc}^{2} / 22$ of Einstein's ordinary quantum relativity energy density from the Lie symmetry group $\mathrm{SO}(10)$ of grand unification of all fundamental forces and without quantum mechanics. American Journal of Mechanics \& Applications, 2(2), 2014, pp. 6-9.

[32] M.S. El Naschie, Cosserat-Cartan modification of Einstein-Riemann relativity and cosmic dark energy density. American Journal of Modern Physics, 3(2), 2014 ,pp. 82-87.

[33] M.S. El Naschie, Rindler space derivation of dark energy. Journal of Modern Physics \& Applications, 2014, ID 6.

[34] M.S. El Naschie, Logarithmic running of 't Hooft-Polyakov monopole to dark energy. Int. Journal of High Energy Physics, 1(1), 2014, pp. 1-5.

[35] M.S. El Naschie, Experimentally based theoretical arguments that Unruh's temperature, Hawking's vacuum fluctuation and Rindler's wedge are physically real. American Journal of Modern Physics, 2(6), 2013, pp. 357-361.

[36] M.S. El Naschie, Determining the missing dark energy density of the cosmos from light cone exact relativistic analysis. Journal of Physics, 2(2), 2013, p. 18-23.

[37] L. Marek-Crnjac, Modification of Eistein's $\mathrm{E}=\mathrm{mc}^{2}$ to $\mathrm{E}=$ $\mathrm{mc}^{2} / 22$. American Journal of Modern Physics. 2(5), 2013, pp. 255-263.

[38] M.S. El Naschie, L. Marek-Crnjac et al, Computing the missing dark energy of a clopen universe which is its own multiverse in addition to being both flat and curved. Fractal Spacetime and Noncommutative Geometry in Quantum \& High Enegy Physics, 3(1), 2013, pp. 3-10.

[39] E.J. Copeland, M. Sami and S. Tsujikawa, Dynamics of dark energy. arXiv, hep-th/0603057V3 16 Jun 2006.

[40] R. Penrose, The Road to Reality. Jonathan Cape , London, 2004.

[41] Y. Baryshev and P. Teerikorpi, Discovery of Cosmic Fractals. World Scientific, Singapore, 2002.

[42] L. Nottale, Scale Relativity. Imperial College Press, London, 2011.

[43] L. Amendola and S. Tsujikawa, Dark Energy, Theory and Observation. Cambridge University Press, Cambridge, 2010.

[44] J. Mageuijo and L. Smolin, Lorentz invariance with an invariant energy scale. arXiv hep-th/0112090V2 18 December 2001.

[45] C. Rovelli, Quantum Gravity. Cambridge University Press, Cambridge, 2004.

[46] L. Hardy, Non-locality of two particles without inequalities for almost all entangled states. Physics Rev. Lett., 71(11), 1993, pp. 1665-1668.

[47] D. Mermin, Quantum mysteries refined. American Journal of Physics, 62(10), 1994, pp. 880-887.

[48] G. Ord, M.S. El Naschie and Ji-Huan He (Editors), Fractal spacetime and noncommutative geometry in high energy physics. 2(1), 2012, pp. 1-79. Asian Academic Publishing Ltd., Hong Kong, China.

[49] L. Sigalotti, A. Meijas, The golden mean in special relativity. Chaos, Solitons \& Fractals, 30, 2006, pp. 521-524.
[50] W. Rindler, Relativity. Oxford University Press, Oxford, 2011.

[51] A. Connes, Noncommutative Geometry. Academic Press, San Diego, USA, 1994.

[52] M.S. El Naschie, "A review of E-infinity theory and the mass spectrum of high energy particle physics." Chaos, Solitons \& Fractals, 19(1), 2004, pp. 209-236.

[53] M.S. El Naschie, On the uncertainty of Cantorian geometry and the two-slit experiment. Chaos, Solitons \& Fractals, 9(3), 1998, pp. 517-529.

[54] M.S. El Naschie, Elementary prerequisites for E-infinity (Recommended background readings in nonlinear dynamics, geometry and topology). Chaos, Solitons \& Fractals, 30, No. 3, 2006, pp. 579-605.

[55] M.S. El Naschie, The concepts of E-infinity, An elementary introduction to the Cantorian-fractal theory of quantum physics. Chaos, Solitons \& Fractals, 22(2), 2004, pp. 495-511.

[56] M.S. El Naschie, On the unification of Heterotic strings, M theory and E-infinity theory. Chaos, Solitons \& Fractals, 11(14), 2000, pp. 2397-2408.

[57] M.S. El Naschie, On a class of general theories for high energy particle physics. Chaos, Solitons \& Fractals, 14(4), 2002, pp. 649-668.

[58] M.S. El Naschie, Superstrings, knots and noncommutative geometry in E-infinity space. Int. Journal of Theoretical Physics, 37(12), 1998, pp. 2935-2951.

[59] H. Aref, Chaos Applied to Fluid Mixing. Pergamon Press. 1995.

[60] M.S. El Naschie, A guide to the mathematics of E-infinity Cantorian spacetime theory. Chaos, Solitons \& Fractals, 25(5), 2005, pp. 955-964.

[61] M.S. El Naschie, Quantum mechanics and the possibility of a Cantorian spacetime. Chaos, Solitons \& Fractals, 1(5), 1991, pp. $485-487$.

[62] M.S. El Naschie, Elementary number theory in superstrings, loop quantum mechanics, twistors and E-infinity high energy physics. Chaos, Solitons \& Fractals, 27(2), 2006, pp. 297-330.

[63] M.S. El Naschie, Quantum gravity, Clifford algebras, fuzzy set theory and the fundamental constants of nature. Chaos, Solitons \& Fractals, 20(3), 2004, pp. 437-450.

[64] M.S. El Naschie, On the unification of the fundamental forces and complex time in the E-infinity space. Chaos, Solitons \& Fractals, 11(7), 2000, pp. 1149-1162.

[65] M.S. El Naschie, Wild topology, hyperbolic geometry and fusion algebra of high energy particle physics. Chaos, Solitons \& Fractals, 13(9), 2002, pp. 1935-1945.

[66] M.S. El Naschie, The theory of Cantorian spacetime and high energy particle physics (An informal review). Chaos, Solitons \& Fractals, 41(5), 2009, pp. 2635-2646.

[67] M.S. El Naschie, Time symmetry breaking, duality and Cantorian spacetime. Chaos, Solitons \& Fractals, 7(4), 1996, pp. 499-518. 
[68] M.S. El Naschie, From experimental quantum optics 25(5), 2005, pp. 969-977.

[69] M.S. El Naschie, Quantum gravity from descriptive set theory. Chaos, Solitons \& Fractals, 19(5), 2004, pp. $1339-1344$

[70] M.S. El Naschie, Is quantum space a random Cantor set with a golden mean dimension at the core? Chaos, Solitons \& Fractals, 4(2), 1994, pp. 177-179.

[71] M.S. El Naschie, Topics in the mathematical physics of E-infinity theory. Chaos, Solitons \& Fractals, 30(3), 2006, pp. 656-663.

[72] Mae-Wan Ho, The Story of Phi, Part 1. Science of the Organism. Institute of Science in Society, 03.03.2014. www.i-sis.org.uk.

[73] Mae-Wan Ho, Watching the Daises Grow, The Story of Phi,
Part 2. Science of the Organism. Institute of Science in Society, 10.03.2014. www.i-sis.org.uk.

[74] Mae-Wan Ho, Golden Music of The Brain, The Story of Phi, Part 3. Science of the Organism. Institute of Science in Society, 17.03.2014. www.i-sis.org.uk.

[75] Mae-Wan Ho, Golden Cycles and Organic Spacetime. The Story of Phi, Part 4. Science of the Organism. Institute of Science in Society, 24.03.2014. www.i-sis.org.uk.

[76] Mae-Wan Ho, Golden Geometry of E-infinity Fractal Spacetime. The Story of Phi, Part 5. Science of the Organism. Institute of Science in Society, 31.03.2014. www.i-sis.org.uk.

[77] Mae-Wan Ho, E-infinity Spacetime, Quantum Paradoxes and Quantum Gravity. The Story of Phi, Part 6. Science of the Organism. Institute of Science in Society, 07.04.2014. www.i-sis.org.uk. 